\title{
Renormalization of the weak hadronic current in the nuclear medium
}

\author{
T. Siiskonen* \\ Helsinki Institute of Physics, University of Helsinki, P.O. Box 9, \\ FIN-00014 Helsinki, Finland \\ M. Hjorth-Jensen \\ Department of Physics, University of Oslo, N-0316 Oslo, Norway \\ J. Suhonen \\ Department of Physics, University of Jyväskylä, P.O. Box 35, \\ FIN-40351 Jyväskylä, Finland
}

\begin{abstract}
The renormalization of the weak charge-changing hadronic current as a function of the reaction energy release is studied at the nucleonic level. We have calculated the average quenching factors for each type of current (vector, axial vector and induced pseudoscalar). The obtained quenching in the axial vector part is, at zero momentum transfer, $19 \%$ for the $s d$ shell and $23 \%$ in the $f p$ shell. We have extended the calculations also to heavier systems such as ${ }^{56} \mathrm{Ni}$ and ${ }^{100} \mathrm{Sn}$, where we obtain stronger quenchings, $44 \%$ and $59 \%$, respectively. Gamow-Teller type transitions are discussed, along with the higher order matrix elements. The quenching factors are constant up to roughly 60 $\mathrm{MeV}$ momentum transfer. Therefore the use of energy-independent quenching factors in beta decay is justified. We also found that going beyond the zeroth and first order operators (in inverse nucleon mass) does not give any
\end{abstract}

${ }^{*}$ Contact address: EP Division, CERN, CH-1211 Geneva 23, Switzerland. 
substantial contribution. The extracted renormalization to the ratio $C_{P} / C_{A}$ at $q=100 \mathrm{MeV}$ is $-3.5 \%,-7.1 \%,-28.6 \%$, and $+8.7 \%$ for mass $16,40,56$, and 100 , respectively.

PACS number(s): 21.30.Fe, 23.40.-s, 21.60.Cs, 21.10.Pc

\section{INTRODUCTION}

The phenomenological structure of the weak hadronic current between the proton and neutron states is well determined by its properties under the Lorentz transformation. The additional constraints come from the requirement of time reversal symmetry as well as from the invariance under the $G$-parity transformation (combined charge conjugation and isospin rotation). The resulting interaction Hamiltonian consists of vector (V), axial vector (A), induced weak magnetism $(\mathrm{M})$, and induced pseudoscalar $(\mathrm{P})$ terms together with the associated form factors $C_{\alpha}, \alpha=V, A, M$, or $P$. These form factors are called as coupling constants at zero momentum transfer. The present experimental knowledge does not exclude the presence of the scalar and tensor interactions. However, their contribution is expected to be small due to weak couplings [1].

The values of vector, axial vector, and weak magnetism couplings are well established by beta-decay experiments as well as by the conserved vector current hypothesis (CVC), introduced already in the late 50's [2]. The magnitude of the pseudoscalar coupling is more uncertain, although the partially-conserved axial current hypothesis (PCAC) [3] provides an

estimate along with muon capture experiments in hydrogen [四, 可. The value of $C_{P}$ in the nuclear medium is not precisely established.

In nuclear beta decay, with an energy release up to some $20 \mathrm{MeV}$, only the vector (Fermi) and the axial vector (Gamow-Teller) terms are usually important. The induced pseudoscalar and weak magnetism parts are essentially inactive, since their contributions are proportional to $q / M$, where $q$ is the energy release and $M$ is the nucleon mass (in units where $\hbar=c=1$ ). There are, however, weak nuclear processes, like muon capture, where the energy release is 
much higher (in muon capture, typically $q \approx m_{\mu} \approx 100 \mathrm{MeV}$ ).

Summed theoretical beta decay strengths are systematically larger than the experimental ones. This so-called quenching of the (allowed) decay strength is usually explained in terms of core polarization (degrees of freedom which are left out from the model space) and nonnucleonic degrees of freedom like isobars and meson exchange currents [6]. Many authors (e.g., 6-9]) have established the quenching factors for the Gamow-Teller decays and closely related magnetic dipole $(M 1)$ transitions.

In 10,11] we have self-consistently constructed effective operators for the weak hadronic current between proton and neutron states. These operators, as explained in Sec. III, take into account the above-mentioned core polarization effects, which are expected to be the largest correction to the bare matrix elements [12]. In the present work our aim is to calculate self-consistently the quenching for all types of operators (V, A, M, and P) for energies up to the muon capture range. In addition to the traditional shell-model regimes, $s d$ and $f p$ shells, we have extended our calculations to ${ }_{28}^{56} \mathrm{Ni}$ and ${ }_{50}^{100} \mathrm{Sn}$ as closed-shell cores.

Typically one expects about $20 \%$ quenching of the axial vector part in the $s d$ shell, i.e., the calculated Gamow-Teller matrix elements $\langle\sigma\rangle$ are to be multiplied by a factor $\sim 0.8$ [8] when both core polarization and non-nucleonic degrees are accounted fort. Effects of the same magnitude are expected in the $f p$ shell [9]. The authors in [9] reach the conclusion that the mass dependence of the quenching has saturated already in the $f p$ shell. However, the major shell closures which separate the spin-orbit partners in ${ }^{56} \mathrm{Ni}\left(0 f_{7 / 2}\right.$ and $0 f_{5 / 2}, 0 g_{9 / 2}$ and $\left.0 g_{7 / 2}\right)$ and ${ }^{100} \mathrm{Sn}\left(0 g_{9 / 2}\right.$ and $0 g_{7 / 2}, 0 h_{11 / 2}$ and $\left.0 h_{9 / 2}\right)$ model spaces introduce large first-order corrections to the operators. Thus, the situation is not analogous to the one seen in light nuclei with closed $L S$ shells. For a recent work in the Sn mass region, see e.g., Ref. [13]. We remind the reader that the quenching is always related to the choice of the model space.

The nuclear muon capture process can be used to extract the ratio $C_{P} / C_{A}$. Unfor-

\footnotetext{
${ }^{1}$ The Gamow-Teller strengths $B(\mathrm{GT}) \propto\langle\sigma\rangle^{2}$ are then multiplied by $(0.8)^{2} \approx 0.6$.
} 
tunately, the results for partial capture rates are very sensitive to applied nuclear model, especially to the model space and to the residual two-body interaction (see, e.g., [14 and references therein). The total rates offer perhaps a more reliable source of information, indicating no or only small quenching for the ratio $C_{P} / C_{A}$ [15]. It is of interest to see whether this quenching can be explained in terms of effective charges for the axial vector and pseudoscalar operators, that is, without the complications coming from the nuclear structure calculations.

In addition to the zeroth-order Fermi and Gamow-Teller type operators, our set includes the first-order terms in the transition amplitude (first order in $q / M$ as well as velocitydependent terms). We shall also examine the importance of the second-order terms. We stress that the results obtained in this work can be applied quite generally. We have used the muon as an initial bound state lepton, but the results apply to electron capture as well and therefore to beta decay in general (in our calculations, the muon is nothing but a heavy electron!).

In this work, after a short review of the formalism of the semileptonic weak processes in Sec. [1] and effective operators in Sec. [1], we concentrate on the results in Sec. IV. We consider four cases, with ${ }^{16} \mathrm{O},{ }^{40} \mathrm{Ca},{ }^{56} \mathrm{Ni}$, and ${ }^{100} \mathrm{Sn}$ as closed-shell cores.

\section{INVARIANT AMPLITUDE AND SINGLE-PARTICLE OPERATORS}

After the standard nonrelativistic reduction, the semileptonic charge-changing weak process

$$
\lambda_{b}+p \rightarrow \nu_{\lambda}+n
$$

where $\lambda_{b}$ is a bound (anti)lepton in an atomic $1 S$ orbit and $\nu_{\lambda}$ is the corresponding (anti)neutrino, is described by the amplitude

$$
\mathcal{M}^{2}=\sum_{\kappa u}\left|M_{V}(\kappa, u)+M_{A}(\kappa, u)+M_{P}(\kappa, u)\right|^{2}
$$


We take $\lambda_{b}$ to be a muon, with a mass $m_{\mu}=105.658 \mathrm{MeV}$. The form of the effective weak hadronic current used for Eq. (2) is the most general one consistent with the expected $G$ parity symmetry and time reversal symmetry. The functions $M_{\alpha}(\kappa, u)$ include the form factors, transition operators, and angular momentum couplings. Explicitly, the vector part is given by

$$
\begin{aligned}
M_{V}(\kappa, u) / C_{V}\left(q^{2}\right) & =[0 l u] S_{0 u}(\kappa) \delta_{l u}-\frac{1}{M}[1 \bar{l} u p] S_{1 u}^{\prime}(-\kappa) \\
& +\frac{q \sqrt{3}}{2 M}\left\{\sqrt{\frac{\bar{l}+1}{2 \bar{l}+3}}[0 \bar{l}+1 u+] \delta_{\bar{l}+1, u}+\sqrt{\frac{\bar{l}}{2 \bar{l}-1}}[0 \bar{l}-1 u-] \delta_{\bar{l}-1, u}\right\} S_{1 u}^{\prime}(-\kappa) \\
& +\sqrt{\frac{3}{2}} \frac{q}{M}\left(1+\mu_{p}-\mu_{n}\right)\{\sqrt{\bar{l}+1} W(11 u \bar{l}, 1 \bar{l}+1)[1 \bar{l}+1 u+] \\
& +\sqrt{\bar{l}} W(11 u \bar{l}, 1 \bar{l}-1)[1 \bar{l}-1 u-]\} S_{1 u}^{\prime}(-\kappa)
\end{aligned}
$$

We consider the $V$ and $M$ terms together, as suggested by the CVC: $C_{M}=\left(\mu_{p}-\mu_{n}\right) C_{V} / 2 M$, where $\mu_{p}$ and $\mu_{n}$ are the anomalous magnetic moments of the proton and neutron in nuclear magnetons. The Fermi-type term is the first term on the right hand side of Eq. (3). The axial vector part is given by

$$
M_{A}(\kappa, u) / C_{A}\left(q^{2}\right)=-[1 l u] S_{1 u}(\kappa)+\frac{1}{M}[0 \bar{l} u p] \delta_{\bar{l} u} S_{0 u}^{\prime}(-\kappa)-M_{P}(\kappa, u) / C_{P}\left(q^{2}\right)
$$

including the pseudoscalar part

$$
M_{P}(\kappa, u) / C_{P}\left(q^{2}\right)=-\frac{q}{2 \sqrt{3} M}\left\{\sqrt{\frac{\bar{l}+1}{2 \bar{l}+1}}[1 \bar{l}+1 u+]+\sqrt{\frac{\bar{l}}{2 \bar{l}+1}}[1 \bar{l}-1 u-]\right\} \delta_{\bar{l} u} S_{0 u}^{\prime}(-\kappa) .
$$

The Gamow-Teller-type term is the first term on the right hand side of Eq. (4).

In Eqs. (3)-(5), $\kappa$ labels the quantum numbers of the emitted neutrino $\nu_{\lambda}$,

$$
\begin{array}{ll}
\kappa>0: & j=l-\frac{1}{2}, l=\kappa \\
\kappa<0: & j=l+\frac{1}{2}, l=-\kappa-1,
\end{array}
$$

where $l$ and $j$ are the orbital and total angular momentum quantum numbers of $\nu_{\lambda}$. The quantity $\bar{l}$ is given by $l-\operatorname{Sign}(\kappa), W$ are the usual Racah coefficients, and 


$$
\begin{aligned}
S_{k u}(\kappa) & =\sqrt{2(2 j+1)} W(1 / 21 j l, 1 / 2 u), \quad k=1 \\
& =\sqrt{\frac{2 j+1}{2 l+1}}, \quad k=0 \\
S_{k u}^{\prime}(-\kappa) & =\operatorname{Sign}(\kappa) S_{k u}(-\kappa) .
\end{aligned}
$$

The most important ingredients, the transition operators, are embedded in the reduced matrix elements $[k w u],[k w u \pm]$, and $[k w u p]$. The quantum numbers labelling the matrix elements are $\mathbf{k}=\mathbf{s}_{\lambda}+\mathbf{s}_{\nu}$, so that $k \equiv|\mathbf{k}|=0$ or 1 , and $u \equiv|\mathbf{u}|=\left|\mathbf{J}_{f}+\mathbf{J}_{i}\right|$ is the tensorial rank of the transition operator. The symbol $w$ is the rank of the spherical harmonics and is therefore related to the parity change. It is given by $w=l$ for $[k w u]$ and $[k w u \pm]$ type matrix elements, and $w=l+1$ or $w=l-1$ for $[k w u p]$ type matrix element ( $k$ and $w$ must be able to couple to $u$ ). The symbol $p$ labels the momentum dependent operators. The matrix elements with the corresponding single-particle operators are listed in Table $\mathbb{E}$. These operators are further multiplied by the radial wave function of the initial state lepton [16]. We have taken into account the large component

$$
G_{\mu}(r)=2\left(\alpha Z m_{\mu}^{\prime}\right)^{3 / 2} e^{-\alpha Z m_{\mu}^{\prime} r}
$$

where $\alpha \approx 1 / 137$ is the fine structure constant and $m_{\mu}^{\prime}$ is the reduced muon mass.

The amplitude (2) can be used for the calculations of muon (or electron) capture rates [14,16]. As mentioned in the previous Section, our aim is to calculate the effective charges (effective form factors) for the vector, axial vector, and pseudoscalar parts of the amplitude, so as to help to understand the differences between calculated and experimental rates and other observables.

For the actual calculations, we divide the reduced nuclear matrix elements into singleparticle and many-body parts,

$$
M_{\alpha}(\kappa, u)=\sum_{p n}\left(n\left\|m_{\alpha}(\kappa, u)\right\| p\right) \frac{\left(J_{f}\left\|\left[a_{n}^{\dagger} \tilde{a}_{p}\right]^{J}\right\| J_{i}\right)}{\sqrt{2 J+1}},
$$

where $n \equiv\left(n_{n}, l_{n}, j_{n}\right)$ and $p \equiv\left(n_{p}, l_{p}, j_{p}\right)$ label the single-particle states. The doubly-barred matrix elements are reduced in the angular momentum space. These reduced single-particle 
matrix elements, which we calculate in the harmonic oscillator basis with the single-particle operators $m_{\alpha}(\kappa, u)$, are constructed as described in the following Section. Further, we have defined

$$
\tilde{a}_{j m}=(-1)^{j+m} a_{j,-m}
$$

As an example, from Eq. (雨), the single-particle matrix element for the axial vector part is

$$
\begin{aligned}
\left(n\left\|m_{A}(\kappa, u)\right\| p\right) & =-C_{A}\left(q^{2}\right)\left(n\left\|O_{1 l u} G_{\mu}(r)\right\| p\right) S_{1 u}(\kappa)+\frac{C_{A}\left(q^{2}\right)}{M}\left(n\left\|O_{0 \bar{l} u p} G_{\mu}(r)\right\| p\right) \delta_{\overline{l u}} S_{0 u}^{\prime}(-\kappa) \\
& +C_{A}\left(q^{2}\right) \frac{q}{2 \sqrt{3} M}\left\{\sqrt{\frac{\bar{l}+1}{2 \bar{l}+1}}\left(n\left\|O_{1 \bar{l}+1 u+} G_{\mu}(r)\right\| p\right)\right. \\
& \left.+\sqrt{\frac{\bar{l}}{2 \bar{l}+1}}\left(n\left\|O_{1 \bar{l}-1 u-} G_{\mu}(r)\right\| p\right)\right\} \delta_{\bar{l} u} S_{0 u}^{\prime}(-\kappa) .
\end{aligned}
$$

A closer look into the Gamow-Teller type matrix element gives

$$
\begin{aligned}
-C_{A}\left(q^{2}\right)\left(n\left\|O_{1 l u} G_{\mu}(r)\right\| p\right) S_{1 u}(\kappa) & =-C_{A}\left(q^{2}\right) i^{l_{p}-l_{n}}\left(l_{n} j_{n}\left\|Y_{1 l u}(\hat{\mathbf{r}}, \sigma)\right\| l_{p} j_{p}\right) \\
& \times \int_{0}^{\infty} r^{2} R_{n_{n} l_{n}}(r) j_{l}(q r) R_{n_{p} l_{p}} G_{\mu}(r) d r \sqrt{2(2 j+1)} W(1 / 21 j l, 1 / 2 u) .
\end{aligned}
$$

Here $R_{n l}(r)$ are the radial single-particle wave functions. The reduced matrix element of the vector spherical harmonics is given by

$$
\begin{aligned}
& \left(l_{n} j_{n}\left\|Y_{1 l u}(\hat{\mathbf{r}}, \sigma)\right\| l_{p} j_{p}\right)=\sqrt{\frac{3}{16 \pi^{2}}}(-1)^{l_{p}+j_{p}+j_{n}+l+1} \hat{l} \hat{u} \hat{j}_{p} \hat{j}_{n}\left(\begin{array}{ccc}
j_{p} & j_{n} & u \\
1 / 2 & -1 / 2 & 0
\end{array}\right) \\
& \times\left[\frac{\hat{j}_{n}^{2}+(-1)^{j_{n}+j_{p}+u} \hat{j}_{p}^{2}}{\sqrt{2 u(u+1)}}\left(\begin{array}{ccc}
u & 1 & l \\
1 & -1 & 0
\end{array}\right)+(-1)^{l_{n}+1 / 2+j_{n}}\left(\begin{array}{ccc}
u & 1 & l \\
0 & 0 & 0
\end{array}\right)\right],
\end{aligned}
$$

where $\hat{x}=\sqrt{2 x+1}$. The expressions for the vector and pseudoscalar parts are obtained in a similar way using Eqs. (3) and (5) and Table \&. where the operators $O$ of Eq. (12) are given. For more details, see [16].

The many-body part, which includes the one-body transition density (OBTD), is given by the adopted nuclear model. The effective operators introduced in Sec. III do not affect 
the OBTD, which are taken to be given numbers. We do not calculate them here (see, e.g., 14] for examples). In what follows we shall consider only the single-particle part of Eq. (10). This is analogous to, e.g., M1-transitions, where the effective charges are calculated without referring to the many-body part.

\section{PERTURBATIVE METHODS AND EFFECTIVE OPERATORS TO SECOND ORDER}

In order to derive a microscopic approach to the effective operator within the framework of perturbation theory, we need to introduce various notations and definitions pertinent to the methods exposed. In this section we briefly review how to calculate an effective onebody operator within the framework of degenerate Rayleigh-Schrödinger (RS) perturbation theory [17,18], see also Refs. [6, 12] for a detailed discussion on various effective operator diagrams.

It is common practice in perturbation theory to reduce the infinitely many degrees of freedom of the Hilbert space to those represented by a physically motivated subspace, the model space. In such truncations of the Hilbert space, the notions of a projection operator $P$ onto the model space and its complement $Q$ are introduced. The projection operators defining the model and excluded spaces are defined by

$$
P=\sum_{i=1}^{D}\left|\Phi_{i}\right\rangle\left\langle\Phi_{i}\right|,
$$

and

$$
Q=\sum_{i=D+1}^{\infty}\left|\Phi_{i}\right\rangle\left\langle\Phi_{i}\right|,
$$

with $D$ being the dimension of the model space, and $P Q=0, P^{2}=P, Q^{2}=Q$, and $P+Q=I$. The wave functions $\left|\Phi_{i}\right\rangle$ are eigenfunctions of the unperturbed hamiltonian $H_{0}=T+U$, where $T$ is the kinetic energy and $U$ an appropriately chosen one-body potential,

that of the harmonic oscillator (h.o.) in this calculation. The full Hamiltonian is then 
rewritten as $H=H_{0}+H_{1}$ with $H_{1}=V-U, V$ being e.g., the nucleon-nucleon (NN) interaction or the $G$-matrix to be discussed below. The eigenvalues and eigenfunctions of the full Hamiltonian are denoted by $\left|\Psi_{\alpha}\right\rangle$ and $E_{\alpha}$, i.e.,

$$
H\left|\Psi_{\alpha}\right\rangle=E_{\alpha}\left|\Psi_{\alpha}\right\rangle
$$

Rather than solving the full Schrödinger equation above, one defines an effective Hamiltonian acting within the model space such that

$$
P H_{\mathrm{eff}} P\left|\Psi_{\alpha}\right\rangle=E_{\alpha} P\left|\Psi_{\alpha}\right\rangle=E_{\alpha}\left|\Phi_{\alpha}\right\rangle
$$

where $\left|\Phi_{\alpha}\right\rangle=P\left|\Psi_{\alpha}\right\rangle$ is the projection of the full wave function onto the model space, the model space wave function. In RS perturbation theory, the effective interaction $H_{\text {eff }}$ can be written out order by order in the interaction $H_{1}$ as

$$
P H_{\mathrm{eff}} P=P H_{1} P+P H_{1} \frac{Q}{e} H_{1} P+P H_{1} \frac{Q}{e} H_{1} \frac{Q}{e} H_{1} P+\ldots
$$

Here we have defined $e=\omega-H_{0}$, where $\omega$ is the so-called starting energy, defined as the unperturbed energy of the interacting particles. Similarly, the exact wave function $\left|\Psi_{\alpha}\right\rangle$ can now be written in terms of the model space wave function as

$$
\left|\Psi_{\alpha}\right\rangle=\left|\Phi_{\alpha}\right\rangle+\frac{Q}{e} H_{1}\left|\Phi_{\alpha}\right\rangle+\frac{Q}{e} H_{1} \frac{Q}{e} H_{1}\left|\Phi_{\alpha}\right\rangle+\ldots
$$

In studies of nuclear transitions such as beta decay, the quantity of interest is the transition matrix element between an initial state $\left|\Psi_{i}\right\rangle$ and a final state $\left|\Psi_{f}\right\rangle$ of an operator $\mathcal{O}$ defined as

$$
\mathcal{O}_{f i}=\frac{\left\langle\Psi_{f}|\mathcal{O}| \Psi_{i}\right\rangle}{\sqrt{\left\langle\Psi_{f} \mid \Psi_{f}\right\rangle\left\langle\Psi_{i} \mid \Psi_{i}\right\rangle}}
$$

Since we perform our calculation in a reduced space, the exact wave functions $\left|\Psi_{f, i}\right\rangle$ are not known, only their projections onto the model space. We are then confronted with the problem of how to evaluate $\mathcal{O}_{f i}$ when only the model space wave functions are known. In treating this problem, it is usual to introduce an effective operator $\mathcal{O}_{f i}^{\text {eff }}$, defined by requiring 


$$
\mathcal{O}_{f i}=\left\langle\Phi_{f}\left|\mathcal{O}_{\text {eff }}\right| \Phi_{i}\right\rangle
$$

Observe that $\mathcal{O}_{\text {eff }}$ is different from the original operator $\mathcal{O}_{f i}$. The standard empirical procedure is then to introduce some adjustable parameters in $\mathcal{O}_{f i}^{\text {eff }}$.

The perturbative expansion for the effective operator can then be written in a much similar way as Eqs. (19) and (20), i.e.,

$$
\left\langle\Psi_{f}|\mathcal{O}| \Psi_{i}\right\rangle=\left\langle\Phi_{f}|\mathcal{O}| \Phi_{i}\right\rangle+\left\langle\Phi_{f}\left|\mathcal{O} \frac{Q}{e} H_{1}\right| \Phi_{i}\right\rangle+\left\langle\Phi_{f}\left|\frac{Q}{e} H_{1} \mathcal{O}\right| \Phi_{i}\right\rangle+\left\langle\Phi_{f}\left|\mathcal{O} \frac{Q}{e} H_{1} \frac{Q}{e} H_{1}\right| \Phi_{i}\right\rangle+\ldots
$$

In Fig. 1 we list all diagrams (except folded diagrams) to second order in the interaction evaluated in this work. We do not include Hartree-Fock insertions. For pure Gamow-Teller or Fermi like operators, see e.g., the review article by Towner [6], such diagrams are exactly zero. Another feature of e.g., the Gamow-Teller-type operators is that for several diagrams involving particle-hole contributions, these diagrams are exactly zero unless the particle-hole orbits are spin-orbit partners. This means that for $L S$-closed shell nuclei like ${ }^{16} \mathrm{O}$ and ${ }^{40} \mathrm{Ca}$ diagrams like (I)-(VIII) or (XIII)-(XX) are all zero. However, this picture changes when we move to closed-shell nuclei like ${ }^{56} \mathrm{Ni}$ and ${ }^{100} \mathrm{Sn}$. For $\mathrm{Ni}$ the last proton and neutron holes are in the $0 f_{7 / 2}$ single-particle orbit. This means that the $0 f_{7 / 2}$ hole and the $0 f_{5 / 2}$ particle states in ${ }^{56} \mathrm{Ni}$ yield non-vanishing contributions to the Gamow-Teller-type operator from the above-mentioned diagrams. Similarly, in Sn these contributions are represented by the spinorbit partners in the the $0 g_{9 / 2}$ hole and the $0 g_{7 / 2}$ particle states. These spin-orbit partners yield then $1 \hbar \omega$ intermediates states. Similarly, we have also spin-orbit partners for particles states outside the model space. These are $0 g_{9 / 2}$ and $0 g_{7 / 2}$ for ${ }^{56} \mathrm{Ni}$ and $0 h_{11 / 2}$ and $0 h_{9 / 2}$ for ${ }^{100} \mathrm{Sn}$. These $1 \hbar \omega$ intermediates states are then responsible for the different quenching of the effective operators in the mass regions of ${ }^{16} \mathrm{O}-{ }^{40} \mathrm{Ca}$ and ${ }^{56} \mathrm{Ni}^{-}{ }^{100} \mathrm{Sn}$, respectively.

We end this section with a discussion of how to construct a $G$-matrix. The $G$-matrix enters in turn our perturbative expansion for the effective operator. As is well know in nuclear physics, the NN potential exhibits a repulsive core, which renders any perturbative 
treatment prohibitive. However, one possible way of overcoming this deficiency is to introduce the reaction matrix $G$, which accounts for short range correlations. The $G$-matrix is defined through

$$
G=V+V \frac{Q}{\omega-Q T Q} G
$$

Here, $\omega$ is the energy of the interacting nucleons in a medium and $V$ is the free NN potential. We have assumed that the energy of the intermediate states can be replaced by the free kinetic spectrum $T$, since these states are predominantly of high excitation energy.

In this work we solve Eq. (24) for finite nuclei by employing a formally exact technique for handling $Q$, originally presented by Tsai and Kuo [19] and discussed in Ref. [20]. Tsai and Kuo employed the matrix identity

$$
Q \frac{1}{Q A Q} Q=\frac{1}{A}-\frac{1}{A} P \frac{1}{P A^{-1} P} P \frac{1}{A}
$$

with $A=\omega-T-V$, to rewrite Eq. (24) as

$$
G=G_{F}+\Delta G
$$

where $G_{F}$ is the free $G$-matrix defined as

$$
G_{F}=V+V \frac{1}{\omega-T} G_{F}
$$

The term $\Delta G$ is a correction term defined entirely within the model space $P$ and given by

$$
\Delta G=-V \frac{1}{A} P \frac{1}{P A^{-1} P} P \frac{1}{A} V .
$$

Employing the definition for the free $G$-matrix of Eq. (27), one can rewrite the latter equation as

$$
\Delta G=-G_{F} \frac{1}{e} P \frac{1}{P\left(e^{-1}+e^{-1} G_{F} e^{-1}\right) P} P \frac{1}{e} G_{F},
$$

with $e=\omega-T$.

We see then that the $G$-matrix for finite nuclei is expressed as the sum of two terms; the first term is the free $G$-matrix with no Pauli corrections included, while the second term 
accounts for medium modifications due to the Pauli principle. The second term can easily be obtained by some simple matrix operations involving the model-space matrix $P$ only.

Finally, in order to calculate the $G$-matrix for the various mass regions, we need to define the relevant model spaces used to define the $P$ and $Q$ operators in the equation for $G$. The oscillator energies $\hbar \Omega$ will be derived from $\hbar \Omega=45 A^{-1 / 3}-25 A^{-2 / 3}$, $A$ being the mass number. This yields $\hbar \Omega=13.9, \hbar \Omega=11.0, \hbar \Omega=10.05$, and $\hbar \Omega=8.5 \mathrm{MeV}$ for $A=16$, $A=40, A=56$, and $A=100$, respectively. We choose the model spaces which are believed, from both experiment and theoretical calculations, to be relevant as a first approximation for calculations of effective interactions and operators in the mass areas from $A=16$ to $A=100$. These are the $0 d_{5 / 2}, 0 d_{3 / 2}$, and $1 s_{1 / 2}$ orbits for $A=16$, the $1 p_{3 / 2}, 1 p_{1 / 2}, 0 f_{7 / 2}$, and $0 f_{5 / 2}$ orbits for nuclei in the mass region of $A=40$, the $1 p_{3 / 2}, 1 p_{1 / 2}, 0 f_{5 / 2}$, and $0 g_{9 / 2}$ orbits for nuclei in the mass region of $A=56$ and the $0 h_{11 / 2}, 0 g_{7 / 2}, 1 d_{5 / 2}, 1 d_{3 / 2}$, and $2 s_{1 / 2}$ orbits for $A=100$. For these systems, the closed-shell cores $\left({ }^{16} \mathrm{O},{ }^{40} \mathrm{Ca},{ }^{56} \mathrm{Ni}\right.$, and $\left.{ }^{100} \mathrm{Sn}\right)$ have equal numbers of protons and neutrons, and the model spaces are the same for both protons and neutrons.

The definition of the Pauli operator for the $G$-matrix can be found in Refs. [20,21], where the so-called double-partitioned scheme has been used. A detailed discussion on the computation of the $G$-matrix can be found in Ref. [21]. This definition means that also the shell above that which defines the model space of the effective interaction, is included in the evaluation of the $G$-matrix. For the $1 s 0 d$-shell, this means that we also include the $1 p 0 f$ shell in the definition of the $P$-operator for the $G$-matrix. As a consequence, we have to include in our perturbation expansion ladder type of diagrams where the allowed intermediate states are those of the $1 p 0 f$-shell. With this prescription, we have to evaluate diagrams X, XIII, XIV, and XXIII-XXVIII in Fig. 11.

In our actual calculation of the various effective operators, we truncate the sum over intermediate states at excitations of $4-8 \hbar \omega$ in oscillator energy. This truncation yields and error of $\sim 1 \%$ in our evaluation of the effective operator. The nucleon-nucleon interaction employed in this work is the CD-Bonn interaction of Machleidt et al. [22]. 


\section{RESULTS}

We consider the transition $0^{+} \rightarrow 1^{+}$, which is of rank $u=1$. In addition, $\kappa=-1$ or $\kappa=2$, corresponding to $j=\frac{1}{2}$ and $l=0$ or $j=\frac{3}{2}$ and $l=2$, respectively. The nuclear matrix elements, allowed by these quantum numbers together with parity conservation, are [101], [121], [101-], [121+], [011p], and $[111 p]$. We remind the reader that the matrix element [101] is closely related to the Gamow-Teller matrix element of the nuclear beta decay, only the radial dependence is more complicated due to the possibility of a larger energy release (see Table (I). Our set includes matrix elements which are classified as forbidden in the nuclear beta decay [16].

In order to discuss the average quenching, we define a factor $\rho_{\alpha}$

$$
\rho_{\alpha}(q) \equiv \rho_{\alpha}=\frac{\sum_{p n}\left|\left(n|| m_{\alpha}^{\mathrm{ren}}(\kappa, u)|| p\right)\right|}{\sum_{p n}\left|\left(n|| m_{\alpha}^{\text {bare }}(\kappa, u)|| p\right)\right|},
$$

where $\alpha=V, A$, or $P$, and "ren" and "bare" refer to renormalized and bare single-particle matrix elements, respectively. The sums run over all the single-particle states included in the model space. We use absolute values in the sums in order to avoid cases where two single-particle matrix elements, with same magnitudes and opposite signs, cancel each other. This kind of cancellations do not easily happen in nuclear structure calculations, since the involved OBTD have different magnitudes (and signs).

We start the discussion with $s d$ shell nuclei. The effective operators are calculated with ${ }_{8}^{16} \mathrm{O}$ as a closed-shell core. The model space is the full $1 s 0 d$ shell. From Fig. 20 we see that the quenching for $V, A$, and $P$ terms remains essentially constant for the whole energy range considered. In particular, at the beta decay energies (below $20 \mathrm{MeV}$ ) all $\kappa=-1$ terms are constant. Moreover, we have $\rho_{A}^{2}(0)=0.81$ which is somewhat larger value than the empirical "universal quenching factor" of Ref. [8], fitted to a large body of beta decay data in the $s d$ shell (we remind the reader that our effective operators do not include the subnucleonic degrees of freedom). We also get a clear renormalization in the vector and pseudoscalar parts of the current. Note that CVC does not apply here, since we are not looking at Fermi transitions, the vector-type contribution comes from the higher-order terms. 
Strictly speaking, our quenching factors are applicable only for one-particle systems, e.g., for ${ }^{17} \mathrm{O}$. In practice, these factors are used for the whole model space, and the (weak) mass dependence is simply left out. In fact, we find that $\rho_{A}^{2}(0)=0.81$ for ${ }_{14}^{28} \mathrm{Si}$ as well (at the oneparticle level). At the end of the model space, effective operators derived for the hole state can also be used. Then, often a larger renormalization compared to the particle operator is seen [6], reflecting the mass dependence.

For ${ }_{20}^{40} \mathrm{Ca}$ as a core, Fig. 3, the overall features are very similar to ${ }^{16} \mathrm{O}$. We get slightly more quenching, $\rho_{A}^{2}(0)=0.77$. This is in line with earlier studies, where only a slightly larger quenching for the $f p$ shell is introduced. Our model space includes all the singleparticle orbits of the $0 f 1 p$ shell. In [9] the authors reach the conclusion that already in the $f p$ shell the quenching factor has reached the large- $A$ limit. This is, indeed, confirmed by our results. We also note the good agreement with the results of Ref. [23], Table 1.

Qualitatively, the saturation of the quenching comes from the similar choice of the model space (complete $0 \hbar \omega$ space) in ${ }^{16} \mathrm{O}$ and ${ }^{40} \mathrm{Ca}$. In both cases, the first-order diagrams give zero contribution. Therefore the second-order contribution, with $1 \hbar \omega$ intermediate excitations, is the most important one, and very similar behaviour is expected and, indeed, seen. The differences can be attributed e.g., to different oscillator parameters and differences in the single-particle orbit structures (in ${ }^{40} \mathrm{Ca}$ more $1 \hbar \omega$ excitations are available). Therefore, whenever the model space includes the whole $0 \hbar \omega$ oscillator shell, a similar quenching is to be expected. The small variations depend on the mass of the closed-shell core.

The model space in which the effective operators are calculated for ${ }_{28}^{56} \mathrm{Ni}$ does not have a closed $L S$ core. Now, the first-order transitions between $f_{7 / 2}, f_{5 / 2}, g_{9 / 2}$, and $g_{7 / 2}$ singleparticle orbits become possible (the situation is analogous to the $M 1$ operator, which is diagonal in orbital angular momentum and spin). This is clearly reflected in the values shown in Fig. 4 . We now have $\rho_{A}^{2}(0)=0.56$. We also remind the reader that the Ikeda sum rule for Gamow-Teller beta decays is not fulfilled in this space.

At mass $A=100$ our space includes the single-particle orbits $0 g_{7 / 2}, 1 d_{5 / 2}, 1 d_{3 / 2}, 2 s_{1 / 2}$, and $0 h_{11 / 2}$ above the $N=Z=50\left({ }_{50}^{100} \mathrm{Sn}\right)$ major shell closure. The spin-orbit partners of 
$0 g_{7 / 2}$ and $0 h_{11 / 2}$ orbits, $0 g_{9 / 2}$ and $0 h_{9 / 2}$, are missing from our model space. As in ${ }^{56} \mathrm{Ni}$, this is seen from Fig. 5: All terms are quenched by a factor which is clearly larger than in lighter nuclei with closed $L S$ shells. In the axial vector part we have now $\rho_{A}^{2}(0)=0.41$, which again stays nearly constant up to tens of MeV's, well beyond the beta decay energy range (see also the discussion in Ref. [13]). The diagonal transitions $g_{7 / 2} \rightarrow g_{7 / 2}$ and $h_{11 / 2} \rightarrow h_{11 / 2}$ are responsible for the kink between $q=80$ and $100 \mathrm{MeV}$.

At the single-particle transition level, the spin-flip matrix elements (e.g., $f_{7 / 2} \rightarrow f_{5 / 2}$ ) are evidently more quenched than the diagonal ones. This feature does not depend on the operator or mass. We further note that the Gamow-Teller type matrix element [101], being by far the dominant axial term, follows very closely the trend of the axial vector part through the whole mass range.

The $\kappa=2$ axial vector terms are shown in Fig. 6. Only the axial vector part is shown, since $V$ and $P$ terms are identical to the case $\kappa=-1$. For $A=16,40$, and 56 a strong quenching is obtained at high momentum transfers. This is mainly caused by the spherical Bessel function $j_{w}(q r)$. Oscillations in $\rho_{A}(q)$ are a sign of the interference between $j_{w}(q r)$ and the radial single-particle wave function. Clearly, a coherent extraction of the renormalization is not as feasible as in the $\kappa=-1$ case.

In the report by Ciechanowicz et al. [24] the meson exchange contribution to the muon capture matrix elements was found to be very small, at least in capture by ${ }^{28} \mathrm{Si}$. This is, however, in contrast with the results for $A=12$ nuclei of Ref. [25]. The quenching of the spin matrix element, essentially the Gamow-Teller matrix element, is expected to be dominated by the core polarization correction [6]. However, our results leave some room for the subnucleonic corrections. For example, in the $s d$ shell, about $50 \%$ of the observed quenching [8] comes from the $\Delta$ isobars, meson exchange currents and more complicated many-body terms. The situation is similar also in the beginning of the $f p$ shell $\left({ }^{40} \mathrm{Ca}\right)$.

The second order terms in the invariant amplitude describing the process (四) are proportional to $M^{-2}$. Therefore one would expect their contribution to be at most a few percent of the first order terms [see Eqs. (3), (4), and (5)]. Indeed this is the case. For example in ${ }^{16} \mathrm{O}$, 
the $r$-dependent single-particle matrix elements which, according to Barabanov [26], should be the dominating second order terms are about order of magnitude smaller than the first order terms. Only seldomly are the magnitudes roughly equal (one case in ${ }^{16} \mathrm{O}$ ). Thus, when the first-order terms are scaled by $M^{-1}$ and second-order terms by $M^{-2}$, the magnitudes behave roughly as $50: 1$, respectively. Their contribution is, in this context, neglible. The same conclusion is reached in [26], where detailed expressions for the matrix elements are given.

The quenching factors $\rho_{A}$ and $\rho_{P}$ can be used to estimate the ratio $C_{P} / C_{A}$. We take

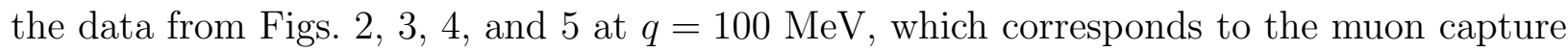
region. Then we have $-3.5 \%,-7.1 \%,-28.6 \%$, and $+8.7 \%$ changes in $C_{P} / C_{A}$ for masses $16,40,56$, and 100 , respectively. If the bare value is taken from $\mathrm{PCAC}, C_{P} / C_{A} \approx 8.4$, we have $C_{P} / C_{A} \approx 8.1,7.8,6.0$, and 9.1 for masses $16,40,56$, and 100 . This yields an average $\sim 7.8$. Although not directly comparable to our results, it is interesting to note the results of Kolbe, Langanke, and Vogel [15]. They used continuum random phase approximation to calculate the part of the capture rate which goes above the particle emission treshold. Their results show a reasonable agreement with data when the bare couplings are used. This nicely demonstrates the fact that if the model space dimension is increased, the couplings should asymptotically reach the bare values.

\section{SUMMARY}

We have constructed the effective transition operators corresponding to the general form of the weak hadronic current between the proton and neutron states. The effects of the renormalization are investigated as a function of the transition $q$-value, and an average over the single-particle transitions is taken separately for vector, axial vector and pseudoscalar terms. We have considered only nucleonic degrees of freedom. In addition to the operators present in the allowed beta decay, we have considered the higher-order corrections to the transition amplitudes. 
In the $s d$ and $f p$ shells, we get $19 \%$ and $23 \%$ quenchings in the axial vector strength, respectively. From these numbers we can conclude that we have reached the large- $A$ limit already in the $f p$ shell, supporting the conclusions of [9]. We have also explained this saturation in qualitative terms. In ${ }^{56} \mathrm{Ni}$ and ${ }^{100} \mathrm{Sn}$, where a major shell closure separates the spin-orbit partners, a larger effect is seen. This is caused by the first order contributions to the effective operator. The quenching stays nearly constant for energies up to some 60 $\mathrm{MeV}$ in all cases. Therefore it is justified to speak about energy-independent quenching factors for beta decays in a given mass region. We also found that the second-order terms (in inverse nucleon mass) are relatively unimportant for most calculations. In particular, the uncertainties in the nuclear model calculations of the one-body transition densities mask these tiny corrections, generally a few percent at maximum.

The quenching factors are used to extract the value of the ratio $C_{P} / C_{A}$ at $q=100 \mathrm{MeV}$. In light systems ${ }^{16} \mathrm{O}$ and ${ }^{40} \mathrm{Ca}$ our results indicate a small (of the order of few percent) quenching. In ${ }^{100} \mathrm{Sn}$, we obtain an enhancement of the same order of magnitude. In ${ }^{56} \mathrm{Ni}$ a large quenching is seen.

The next step in studies of effective operators is the inclusion of subnucleonic degrees of freedom in the evaluation of the different diagrams entering the definition of the effective operator. Especially we have in mind the $\Delta$-isobars as an intermediate state. These states have essentially been neglected due the lack of a suitable $\Delta \Delta$ interaction. We plan to extend our formalism to include such states through the use of a newly refitted nucleonnucleon interaction which includes isobars as explicit degrees of freedom. This interaction [27] accounts for scattering data up to $\sim 1 \mathrm{GeV}$ in laboratory energy. The inclusion of meson exchange effects together with the effective transition operators is also a considerable task, not fully attacked yet.

This work has been supported by the Academy of Finland under the Finnish Centre of Excellence Programme 2000-2005 (Project No. 44875, Nuclear and Condensed Matter Programme at JYFL). 


\section{REFERENCES}

[1] J. Govaerts, Nucl. Instrum. Methods Phys. Res. A402, 303 (1998); Yu. Shitov, Ch. Briancon, V. Brudanin, J. Deutsch, V. Egorov, T. Filipova, J. Govaerts, C. Petitjean, R. Prieels, T. Siiskonen, J. Suhonen, Ts. Vylov, V. Wiaux, I. Yutlandov, and Sh. Zaparov, unpublished.

[2] R. P. Feynman and M. Gell-Mann, Phys. Rev. 109, 193 (1958); S. S. Gershtein and Y. B. Zel'dovich, Sov. Phys. JETP 2, 576 (1956).

[3] M. L. Goldberger and S. B. Treiman, Phys. Rev. 110, 1178 (1958).

[4] G. Bardin, J. Duclos, A. Magnon, J. Martino, A. Richter, E. Zavattini, A. Bertin, M. Piccinini, and A. Vitale, Phys. Lett. B 104, 320 (1981).

[5] D. M. Wright et al., Phys. Rev. C 57, 373 (1998).

[6] I. S. Towner, Phys. Rep. 155, 263 (1987).

[7] B. A. Brown and B. H. Wildenthal, Nucl. Phys. A474, 290 (1987).

[8] B. A. Brown and B. H. Wildenthal, Ann. Rev. Nucl. Part. Sci. 38, 29 (1988).

[9] G. Martinez-Pinedo, A. Poves, E. Caurier, and A. P. Zuker, Phys. Rev. C 53, R2602 (1996).

[10] T. Siiskonen, J. Suhonen, and M. Hjorth-Jensen, Phys. Rev. C 59, R1839 (1999).

[11] T. Siiskonen, J. Suhonen, and M. Hjorth-Jensen, J. Phys. G 25, L55 (1999).

[12] B. Castel and I. S. Towner, Modern Theories of Nuclear Moments (Clarendon Press, Oxford, 1990).

[13] M. Karny et al., Nucl. Phys. A640, 3 (1998).

[14] T. Siiskonen, J. Suhonen, V. A. Kuz'min, and T. V. Tetereva, Nucl. Phys. A635, 446 (1998); ibid, A651, 437 (1999). 
[15] E. Kolbe, K. Langanke, and P. Vogel, Phys. Rev. C 50, 2576 (1994).

[16] M. Morita and A. Fujii, Phys. Rev. 118, 606 (1960).

[17] T. T. S. Kuo and E. Osnes, Folded-Diagram Theory of the Effective Interaction in Atomic Nuclei, Springer Lecture Notes in Physics (Springer, Berlin, 1990), Vol. 364.

[18] I. Lindgren and J. Morrison, Atomic Many-Body Theory (Springer, Berlin, 1985).

[19] S. F. Tsai and T. T. S. Kuo, Phys. Lett. B 39, 427 (1972).

[20] E. M. Krenciglowa, C. L. Kung, T. T. S. Kuo, and E. Osnes, Ann. Phys. (N.Y.) 101, 154 (1976).

[21] M. Hjorth-Jensen, E. Osnes, and T. T. S. Kuo, Phys. Rep. 261, 125 (1995).

[22] R. Machleidt, F. Sammarruca, and Y.Song, Phys. Rev. C 53, R1483 (1996).

[23] I. S. Towner, Phys. Lett. B 333, 13 (1994).

[24] S. Ciechanowicz, F. C. Khanna, and E. Truhlik, in Mesons and Light Nuclei '98, Edited by J. Adam, P. Bydžavský, J. Dobeš, R. Mach, J. Mareš, and M. Sotona (World Scienticic, Singapore, 1999), p. 478.

[25] P. A. M. Guichon and C. Samour, Nucl. Phys. A382, 461 (1982).

[26] A. L. Barabanov, preprint IAE-6119/2 (Kurchatov Institute, Moscow, 1999); Phys. Atom. Nucl. 63, 1187 (2000).

[27] R. Machleidt, private communication. 


\section{TABLES}

TABLE I. Reduced nuclear matrix elements and the corresponding single-particle operators [without the lepton radial wave function $G_{\mu}(r)$ ]. The $j_{w}(q r)$ are the spherical Bessel functions and $Y_{k w u}^{M}$ are the vector spherical harmonics 16]. The momentum operator for nucleons is $\mathbf{p}$, and $p_{\nu}$ is the momentum of the neutrino.

\begin{tabular}{ll}
\hline \hline Matrix element & $O_{k w u}$ \\
\hline$[0 w u]$ & $j_{w}(q r) Y_{0 w u}^{M}(\hat{\mathbf{r}}) \delta_{w u}$ \\
{$[1 w u]$} & $j_{w}(q r) Y_{1 w u}^{M}(\hat{\mathbf{r}}, \sigma)$ \\
\hline & $O_{k w u \pm}$ \\
\hline$[0 w u \pm]$ & {$\left[j_{w}(q r) \pm \alpha Z\left(m_{\mu}^{\prime} / p_{\nu}\right) j_{w \mp 1}(q r)\right] Y_{0 w u}^{M}(\hat{\mathbf{r}}) \delta_{w u}$} \\
{$[1 w u \pm]$} & {$\left[j_{w}(q r) \pm \alpha Z\left(m_{\mu}^{\prime} / p_{\nu}\right) j_{w \mp 1}(q r)\right] Y_{1 w u}^{M}(\hat{\mathbf{r}}, \sigma)$} \\
\hline & $O_{k w u p}$ \\
\hline$[0 w u p]$ & $i j_{w}(q r) Y_{0 w u}^{M}(\hat{\mathbf{r}}) \sigma \cdot \mathbf{p} \delta_{w u}$ \\
\hline $1 w u p]$ & $i j_{w}(q r) Y_{1 w u}^{M}(\hat{\mathbf{r}}, \mathbf{p})$ \\
\hline \hline
\end{tabular}


FIGURES 

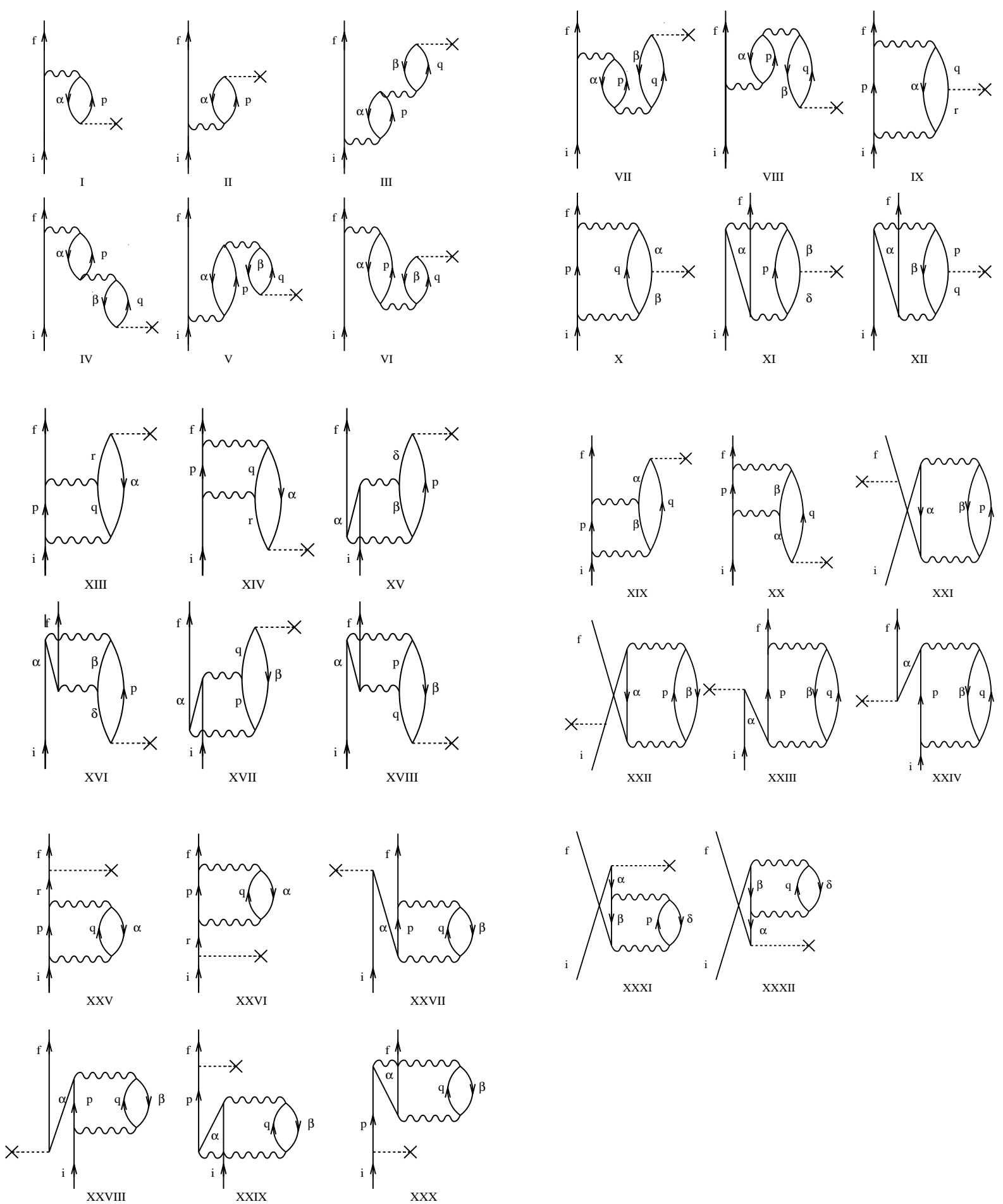
FIG. 1. Non-folded diagrams to second order in the interaction included in the evaluation of the effective operator. Hole states are represented by greek letters while particle states are given by roman letters. The operator itself is given by $---\times$ in the various diagrams, while the wiggly lines are the nuclear $G$-matrix. Folded diagrams to second order in the interaction are included in the calculation but not shown here. 


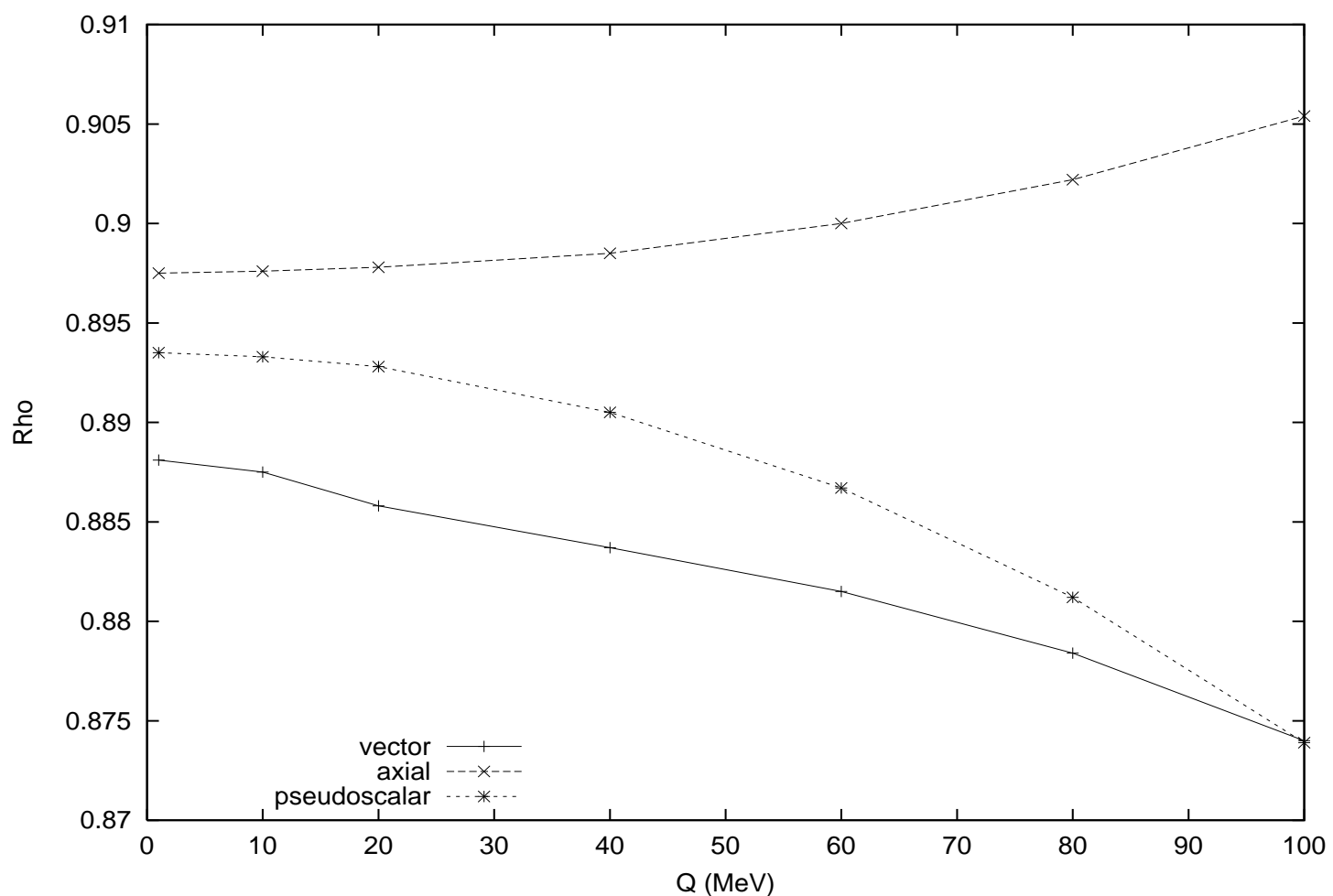

FIG. 2. Renormalization of the vector, axial vector and pseudoscalar terms with ${ }^{16} \mathrm{O}$ as the closed-shell core, $\kappa=-1(J=0 \rightarrow 1$ transition, $\Delta \pi=$ no $)$. 


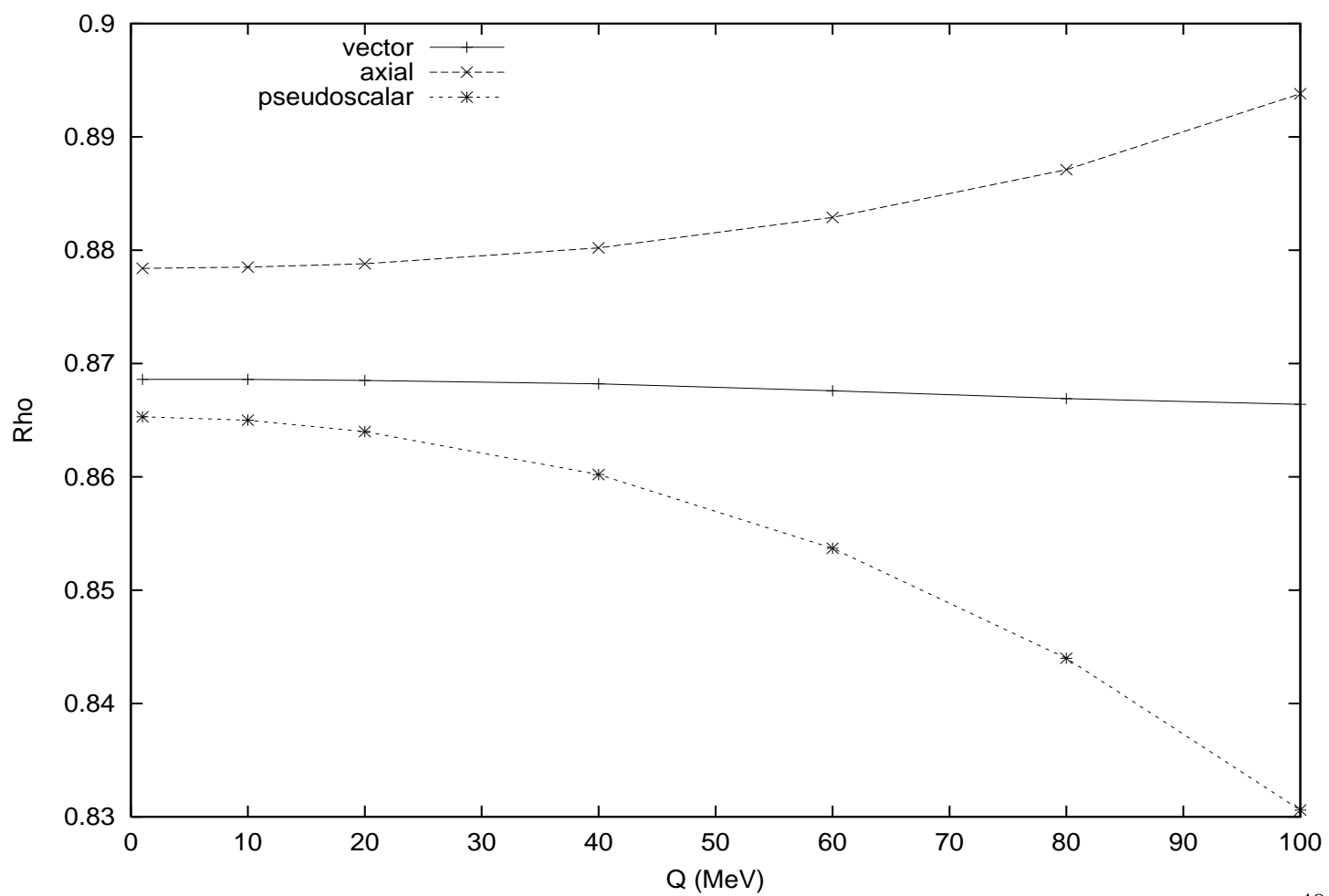

FIG. 3. Renormalization of the vector, axial vector and pseudoscalar terms with ${ }^{40} \mathrm{Ca}$ as the closed-shell core, $\kappa=-1(J=0 \rightarrow 1$ transition, $\Delta \pi=$ no $)$. 


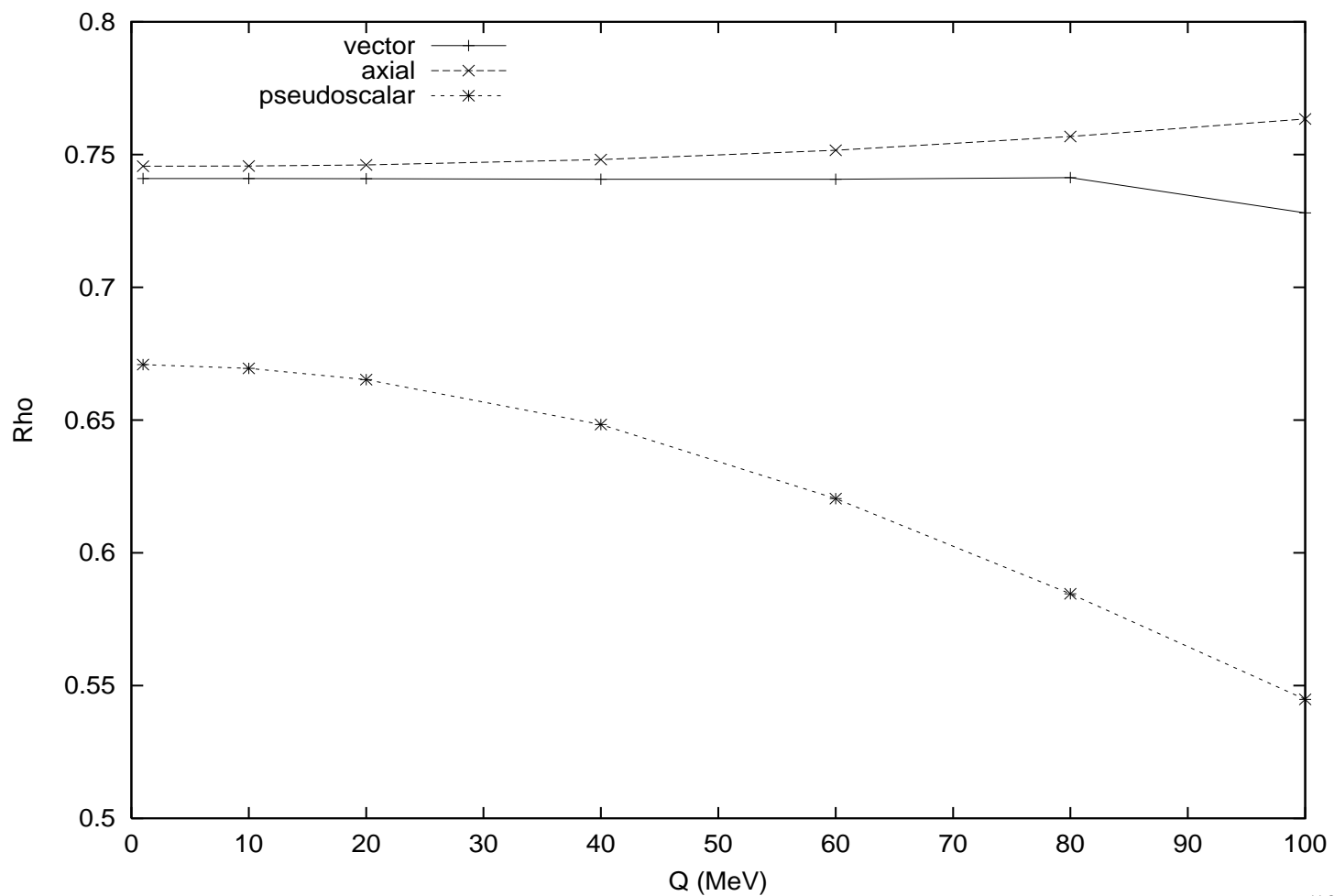

FIG. 4. Renormalization of the vector, axial vector and pseudoscalar terms with ${ }^{56} \mathrm{Ni}$ as the closed-shell core, $\kappa=-1(J=0 \rightarrow 1$ transition, $\Delta \pi=$ no $)$. 


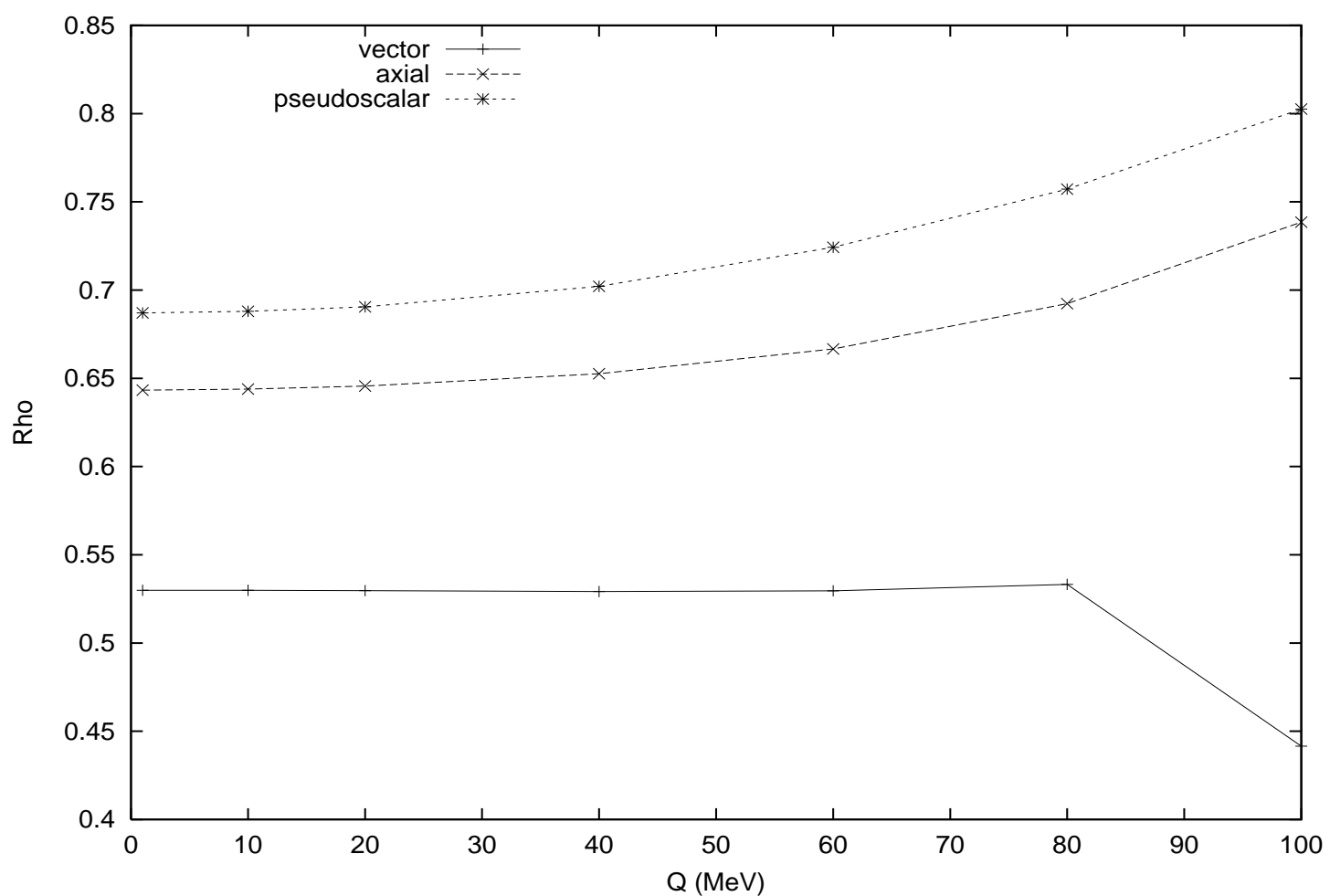

FIG. 5. Renormalization of the vector, axial vector and pseudoscalar terms with ${ }^{100} \mathrm{Sn}$ as the closed-shell, $\kappa=-1(J=0 \rightarrow 1$ transition, $\Delta \pi=$ no $)$. 


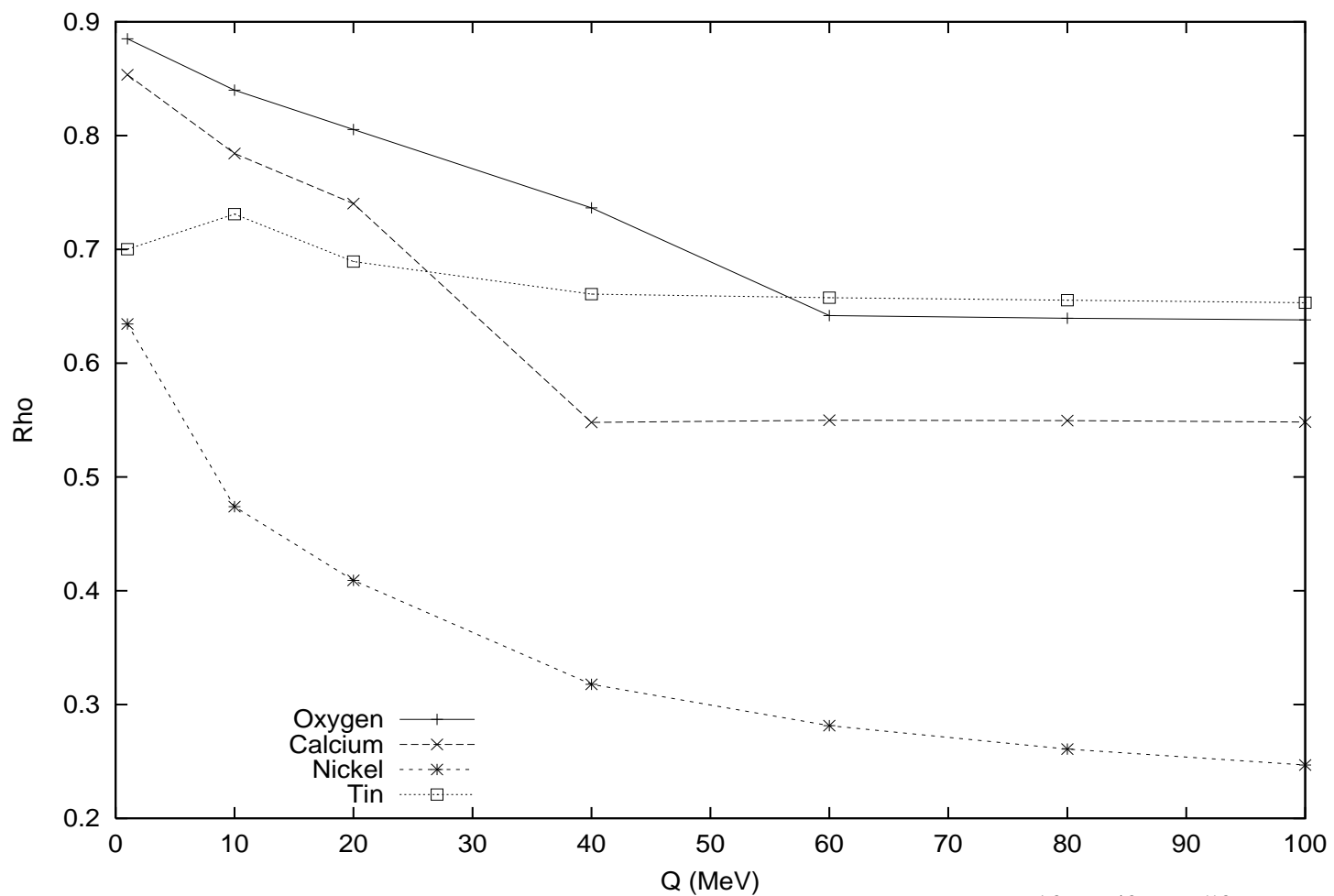

FIG. 6. Renormalization of the axial vector term with $\kappa=2$ for ${ }^{16} \mathrm{O},{ }^{40} \mathrm{Ca},{ }^{56} \mathrm{Ni}$, and ${ }^{100} \mathrm{Sn}$ as closed-shell cores $(J=0 \rightarrow 1$ transition, $\Delta \pi=$ no). 